\title{
Small Angle X-Ray Study of Cellulose in Cotton Using Correlation Functions
}

\author{
T. MisRa, D. K. Bisoyi, T. PATEl, \\ K. C. PATra, and A. PATEL* \\ Department of Physics, Regional Engineering College, \\ Rourkela-769 008, Orissa, India
}

(Received December 7, 1987)

\begin{abstract}
This work shows that the small angle X-ray scattering (SAXS) intensity of dewaxed cotton of local origin at $25^{\circ} \mathrm{C}$ deviates from Porod's law establishing that cotton at $25^{\circ} \mathrm{C}$ falls under non-ideal two-phase structure characterised by continuous variation of electron density at the phase boundary. Therefore the mean square of the electron density gradient $\left\langle|\operatorname{grad} \eta|^{2}\right\rangle$ in isotropic structure is proportional to the fourth moment $\int s^{4} I(s) \mathrm{d} s$, of the SAXS intensity distribution in the reciprocal space and to the second derivative of the correlation function in the origin. The above relation given by Vonk has been used to find the thickness $E$ of the transition region along with the following important physical parameters. They are $D$, the average periodicity transverse to the layer, $S / V$, the specific inner surface, $\phi_{1}$ and $\phi_{2}$, the volume fraction of two phases, i.e., matter and void, $l_{\mathrm{c}}$, the length of coherence, $\bar{l}_{1}$ and $\bar{l}_{2}$, the transversal lengths, $\bar{l}_{\mathrm{r}}$, the range of inhomogeneity and $2 E / D$, the volume fraction of the transition layer. Two values of $E$ have been obtained by the method of Vonk and Ruland. The SAXS intensity data of cotton sample was obtained by a technique using a compact Kratky camera.

KEY WORDS Back Ground Corrected SAXS Intensity / Cellulose / Width of Transition Layer / Correlation Functions / Transverse Periodicity /
\end{abstract}

Cotton as a natural fibre finds extensive use in day to day life. Cotton fibres, like most vegetable fibres are essentially cellulosic in nature and the percentage of cellulose is as high as $96 \%$ in unpurified cotton. The other components of cotton are protein $1.1-1.9 \%$, pectic substance $0.7-1.2 \%$, ash $0.7-1.6 \%$, wax $0.4-1.0 \%$ and few other trace materials. However purified cotton contains $99.7 \%$ pure cellulose. ${ }^{4}$ The cellulose in cotton is in the form of fibrils which spiral at an angle to the length axis of the cell. It is arranged in several ordered levels of orientation in the fibre. At the molecular level, the long molecules are shown by Xray diffraction to be arranged into highly ordered crystalline regions. X-Ray diffraction is considered the basic method for the structural studies of polymer solids, because of direct relationship between the diffraction pattern and order. ${ }^{5}$

As cotton is cellulosic in nature and comes under macromolecular system, a small angle X-ray scattering (SAXS) study has been undertaken to throw some light on its macromolecular structure.

Small angle X-ray scattering takes place in systems having macromolecular structure. The electron density difference between the two phases of the system, which occurs over a distance of 100 to $1000 \AA$, causes small angle $\mathrm{X}$-ray scattering. This phenomenon was first observed by Krishnamurti, ${ }^{6}$ Mark, ${ }^{7}$ and

* Present address: Department of Chemistry and Chemical Engineering, Steven Institute of Technology, Hoboken, New Jersey 07030, U.S.A. 
Hendricks $^{8}$ around 1930. Porod's laws 9,10 were applied to obtain physical parameters of densely packed system for an ideal two-phase structure having sharply defined phase boundaries, where it was observed that the product $\tilde{I}(\theta) \cdot \theta^{3}$ comes out to be a constant at a large scattering angle. Here, $\theta$ is half the scattering angle and $\tilde{I}$ is the smeared-out intensity. However, in some cases the above product decreases gradually with $\theta$. This was explained by Vonk, ${ }^{1}$ as due to the fact that the electron density does not change abruptly, but changes gradually over certain range between twophases. This range is called the width of the transition layer $E$. Such a system is known as a non-ideal two-phase system. This model of non-ideal two-phase system can be considered as one in which the hard core is coated with a soft sheath with a continuously varying electron density.

\section{EXPERIMENTAL}

\section{Material}

This sample of cotton of local origin was first combed to make the fibrils parallel. The fibres were tied into small bundles and were cooked in a $(1: 2)$ mixture of alcohol and benzene under slow and gradual rise and fall of temperature of the bath from $30^{\circ} \mathrm{C}$ to $55^{\circ} \mathrm{C}$ as done by Roy. ${ }^{11}$ The process of heating and cooling was done every two hours for a period of $12 \mathrm{~h}$. Finally the fibre was removed from the mixture at a temperature of $29^{\circ} \mathrm{C}^{12}$ As a result of this treatment the specimen attains a "hohlraum" character (according to Porod ${ }^{13}$ and Ratho et al. ${ }^{14}$ ), i.e., the substance occurs in layers like the pages of the book with free spaces in between. This is taken as a dewaxed purified sample of cotton.

\section{$X$-Ray Measurement}

The small angle $\mathrm{X}$-ray scattering measurements were made on a Kratsky camera ${ }^{3}$ using an entrance slit of $150 \mu \mathrm{m}$ and a counter slit of $375 \mu \mathrm{m}$ to a resolution of about $540 \AA$ (Figure 1). X-Ray radiations were produced by a highly stabilized Siemens Kristalloflex 4 generator and Siemens $\mathrm{AgCu}$ 40/2 Copper target tube operated at $50 \mathrm{kV}$ and $30 \mathrm{~mA}$ and the monochromatization was achieved using a $9 \mu \mathrm{m}$ thick Ni filter. Further monochromatization was achieved with a pulse height discriminator to eliminate the effect of $\mathrm{Cu}-K_{\beta}$ radiation and the sample was irradicated with $\mathrm{Cu}-K_{\alpha}(\lambda=1.54 \AA)$. To reduce the scattering of the X-rays by air between the sample and detector, the vacuum chamber in the intervening space was maintained at a pressure between 1 to 2 Torr. The dewaxed sample was inserted in a Mark capillary tube of diameter $0.1 \mathrm{~cm}$ and the capillary was aligned parallel to

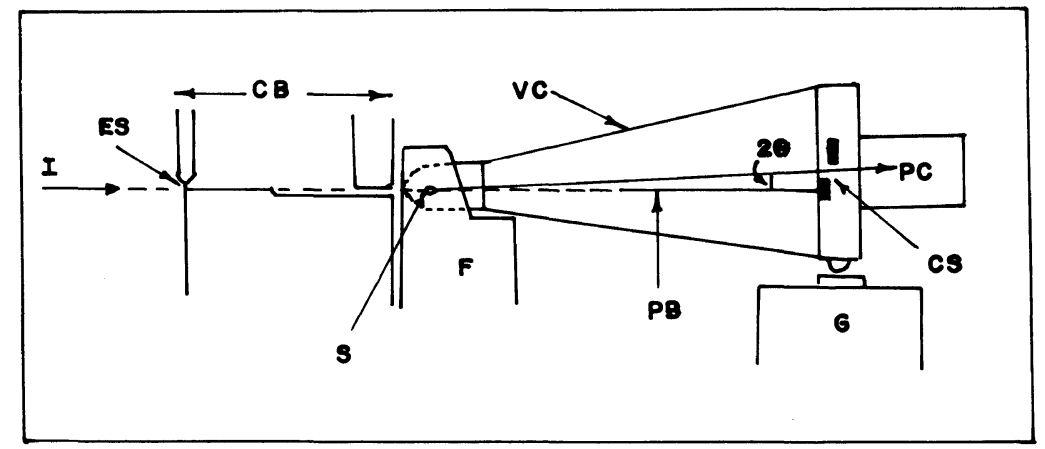

Figure 1. Schematic of the kratky camera not drawn to scale. Adapted from ref 3: I, incident X-ray beam; ES, entrance slit; CB, collimation block; S, sample; F, fork; VC, vacuum chamber; PB, primary beam; G, electronic goniometer; CS, counter slit; PC, proportional counter. Cotton $-25^{\circ} \mathrm{C}$ 
the length of the primary beam. The sample was maintained at $25^{\circ} \mathrm{C}$ by a constant temperature device supplied by Anton Paar K. G. Austria. The X-ray intensity was measured by a Siemen type B proportional counter operated between $1790-1800$ volts supplied by a stabilized high voltage unit and intensity was recorded in a fixed pulse mode. The angle of scattering for the sample was from $2.9 \times 10^{-3}$ to $28.3 \times 10^{-3}$ radians. The distance of the sample from the counter-slit, a, was kept at $27.5 \mathrm{~cm}$.

\section{THEORY}

It has been shown by $\operatorname{Vonk}^{1}$ that, for a general two-phase system having isotropic structure, the relation

$$
16 \pi^{3} \int_{0}^{\infty} s^{4} I_{\mathrm{a}}(s) \mathrm{d} s=\int_{0}^{\infty}|\operatorname{grad} \eta|^{2} \mathrm{~d} v_{\mathrm{r}}
$$

holds good, where $I_{\mathrm{a}}(s)$ is the desmeared intensity in absolute unit, $\eta$ is the deviation of electron density of the sample at any point from the mean value and $s$ is the co-ordinate in the reciprocal or Fourier space given by the relation $s=2 \theta / \lambda$, where $2 \theta$, the scattering angle, is equal to $x / a$. Here $x$ is the position coordinate of scattered intensity from the centre of the primary beam.

The above equation can be regarded as parallel to the well known relation

$$
4 \pi \int_{0}^{\infty} s^{2} I_{\mathrm{a}}(s) \mathrm{d} s=\int_{0}^{\infty} \eta^{2} \mathrm{~d} v_{\mathrm{r}}
$$

where $\int_{0}^{\infty} s^{2} I_{\mathrm{a}}(s) \mathrm{d} s$ is generally known as the invariant.

If absolute intensities are not available, a very useful parameter $R$, given by the ratio of (1) and (2) can be obtained as

$$
R=\frac{\left\langle|\operatorname{grad} \eta|^{2}\right\rangle}{\left\langle\eta^{2}\right\rangle}=4 \pi^{2} \cdot \frac{\int_{0}^{\infty} s^{4} I_{\mathrm{a}}(s) \mathrm{d} s}{\int_{0}^{\infty} s^{2} I_{\mathrm{a}}(s) \mathrm{d} s}
$$

$$
=6 \pi^{2} \frac{\int_{0}^{\infty} s^{3} \tilde{I}(s) \mathrm{d} s}{\int_{0}^{\infty} s \tilde{I}(s) \mathrm{d} s}
$$

Here $I(s)$ and $\tilde{I}(s)$ are the desmeared and smeared-out intensities, respectively, in arbitrary units.

The ratio $R$ is a useful parameter for characterization of structure. In any ideal two-phase structure, the gradient at the phase boundary is infinite and consequently $R$ also goes to infinity. On the other hand if $R$ is finite the electron density changes from one phase to the other continuously over a region known as the transition layer of width or thickness $E$. The above equation has been transformed to variable $x$ by Misra, Patra, and Patel ${ }^{15}$ as

$$
R=\frac{3}{2}\left(\frac{2 \pi}{\lambda a}\right)^{2} \int_{0}^{\infty} x^{3} \tilde{I}(x) \mathrm{d} x / \int_{0}^{\infty} x \tilde{I}(x) \mathrm{d} x
$$

The value of $R$ obtained from (4) determines the nature of the sample, as to whether it belongs to an ideal or non-ideal two-phase system.

The value of $E$ can be obtained from $C(r)$, the three-dimensional correlation function of a sample normalized to unity at the origin, in real space. The equation connecting $E$ and $C(r)$ has been given by Vonk ${ }^{1}$ as

$$
E=\frac{-4}{R}\left(\frac{\mathrm{d} C(r)}{\mathrm{d} r}\right)_{r=E}
$$

In order to obtain $E$, it is required to evaluate the values of $C(r)$ at various values of $r$ in real space. It has been shown by Mering and Tchoubar $^{16}$ that $C(r)$ can be calculated from the expression

$$
C(r)=\int_{0}^{\infty} s \tilde{I}(s) J_{0}(2 \pi r s) \mathrm{d} s / \int_{0}^{\infty} s \tilde{I}(s) \mathrm{d} s
$$

where $J_{0}$ is the Bessel function of zero order of the first kind. In the above expression, the three-dimensional correlation function $C(r)$ is determined from the smeared-out intensity $\tilde{I}(s)$, where the slit correction for infinite height 
given by the relation,

$$
\tilde{I}=\int_{-\infty}^{+\infty} I\left(\sqrt{s^{2}+t^{2}}\right) \mathrm{d} t
$$

is incorporated ${ }^{17}$ and contained in the Bessel function $J_{0}$. Here ' $t$ ' is an arbitrary variable representing the slit height and $C(r)$ has been written in terms of $x$ by Misra et al. ${ }^{15}$ and given by:

$$
C(r)=\int_{0}^{\infty} x \tilde{I}(x) J_{0}(2 \pi r x / \lambda a) \mathrm{d} x / \int_{0}^{\infty} x \tilde{I}(x) \mathrm{d} x
$$

For a layer structure, Kortleve and Vonk ${ }^{18}$ have used the one-dimensional correlation function $C_{1}(y)$. Mering and Tchoubar ${ }^{16}$ have shown that

$$
C_{1}(y)=\frac{\int_{0}^{\infty} s \tilde{I}(s)\left[J_{0}(Z)-Z J_{1}(Z)\right] \mathrm{d} s}{\int_{0}^{\infty} s I(s) \mathrm{d} s}
$$

Here $Z=2 \pi s y$ and $J_{1}$ is the Bessel function of first order of the first kind. When changed to the $x$ variable, the above equation (Misra $e t$ al. ${ }^{15}$ ) reduces to

$$
C_{1}(y)=\frac{\int_{0}^{\infty} x \tilde{I}(x)\left[J_{0}(Z)-Z J_{1}(Z)\right] \mathrm{d} x}{\int_{0}^{\infty} x \tilde{I}(x) \mathrm{d} x}
$$

where $Z=2 \pi x y / \lambda a$. It was shown by Vonk ${ }^{1}$ that the position of the first subsidiary maximum in the one-dimensional correlation function gives the value of the average periodicity $D$ transverse to the layers. The value of $D$ can be obtained by picking up the position of the first subsidiary maximum in $C_{1}(y)$ vs. $y$ curve.

The equation:

$$
\left[\mathrm{d} C_{1}(y) / \mathrm{d} y\right]_{y>E}=-\frac{1}{D}(\Delta \eta)^{2} /\left\langle\eta^{2}\right\rangle
$$

derived by Vonk ${ }^{1}$ can be used to calculate the value of $(\Delta \eta)^{2} /\left\langle\eta^{2}\right\rangle$, where $\Delta \eta$ is the electron density difference between the two phases.
Here, the slope is taken at a point where $y$ is greater than $E$.

It has been proved by Vonk ${ }^{1}$ that the second differentials of $C(r)$ and $C_{1}(y)$ at the origin are given by the following relation

$$
\left[\frac{\mathrm{d}^{2} C_{1}(y)}{\mathrm{d} y^{2}}\right]_{y=0}=3\left[\frac{\mathrm{d}^{2} C(r)}{\mathrm{d} r^{2}}\right]_{r=0}
$$

For a layer structure, as shown by Vonk ${ }^{1}$ the specific inner surface, $S / V$ defined as the phase boundary per unit volume of the dispersed phase, can be written as

$$
\frac{S}{V}=\frac{2}{D}
$$

For a non-ideal two-phase structure, the following relation holds good $\left(\right.$ Vonk $\left.^{1}\right)$.

$$
\frac{\left\langle\eta^{2}\right\rangle}{(\Delta \eta)^{2}}=\left(\phi_{1} \phi_{2}-\frac{E}{6} \cdot \frac{S}{V}\right)
$$

Here $\phi_{1}$ and $\phi_{2}$ are the volume fractions of the two phases, matter and void regions, respectively. For this, the phase boundary is shown at the middle of the transition layer. Assuming the sum of the volume fractions of both phases to be unity, the values of $\phi_{1}$ and $\phi_{2}$ can be determined from the above relation.

For a two-phase system, an idea of the statistical distribution of crystalline (matter) and void regions can be formed by shooting arrows in all possible directions. The average lengths of the arrows in the two phases are called the transversal lengths $\bar{l}_{1}$ and $\bar{l}_{2}$. They are give by Mittelbach and Porod ${ }^{19}$ as

$$
\bar{l}_{1}=4 \phi_{1} V / s \text { and } \bar{l}_{2}=4 \phi_{2} V / s
$$

with the range of inhomogeneity $\bar{l}_{\mathrm{r}}$ as

$$
\frac{1}{\bar{l}_{\mathrm{r}}}=\frac{1}{\bar{l}_{1}}+\frac{1}{\bar{l}_{2}}
$$

The length of coherence for a specimen as given by above workers is

$$
l_{\mathrm{c}}=2 \int_{0}^{\infty} C(r) \mathrm{d} r
$$

Another method for estimating the value of 
$E$ has been suggested by Ruland. ${ }^{2}$

The functional relationship of $\tilde{I}(s)$ with $s$ at the tail end of the SAXS pattern, for non-ideal two-phase system is given by

$$
\tilde{I}(s \rightarrow \infty)=\frac{\pi c}{2}\left(\frac{1}{s^{3}}-2 \pi^{2} E^{2} / 3 s\right)
$$

Here $c$ is the proportionality constnat. For an ideal two-phase structure, $E=0$ and hence the above equation reduces to Porod's law. The above equation when transformed to $x$ takes the form

$$
\tilde{I}(x \rightarrow \infty) \cdot x=\frac{\pi c(\lambda a)^{3}}{2} \cdot x^{-2}-\frac{\pi^{3} c}{3}(\lambda a) E^{2}
$$

The value of $E$ can be calculated from the graph of $\tilde{I}(x \rightarrow \infty)$. $x$ versus $x^{-2}$ known as Ruland plot.

\section{BACKGROUND CORRECTION}

In no experimental set up it is possible to record the SAXS pattern of the sample alone. A continuous background scattering is always mixed with the SAXS pattern of the experimental sample. (It is therefore necessary to apply the background correction to estimate the effect of continuous background scattering:) It was found that estimation of the width of transition layer by the method of Vonk ${ }^{1}$ and Ruland $^{2}$ is sensitive to the systematic error in the tail region of the SAXS curve. Moreover, the correlation function at the origin is also sensitive to the error in the tail region of SAXS curve $\left(\right.$ Vonk $\left.^{1}\right)$. Therefore special care must be taken to separate the SAXS intensity $\tilde{I}(s)$ from the continuous background scattering $\tilde{I}_{\mathrm{bg}}(s)$ $\left(\right.$ Vonk $\left.^{1}\right)$ as reported by Kortleve et al. ${ }^{20}$

The continuous background in some pattern shows an upward trend at large values of $s$ after a minimum is attained in the observed SAXS curve. In such cases the experimentally observed data at the tail end can be fitted to an equation of the type

$$
\tilde{I}_{\mathrm{bg}}(s)=a+b s^{n}
$$

where $a$ and $b$ are constants and $n$ is an even integer between 0 and 10 .

\section{CALCULATIONS AND RESULTS}

The experimental SAXS pattern for cotton at $25^{\circ} \mathrm{C}$ (Figure 2) attains a minimum value at $x=0.58 \mathrm{~cm}$ and then shows an upward trend. In Figure 2 the scattering points from $x=0.25$ to $x=0.78$ are shown on a magnified scale. Therefore the observed data at the tail end were fitted to an equation of the type 15 and the values of $a, b$, and $n$ were obtained as $4.9,7.8$, and 6 , respectively. Using the values of $a, b$, and $n$ the background correction for each and every point of the experimental curve upto $x=0$ were obtained and are also shown in Figure 2, as deducted from the observed experimental intensities. The background corrected SAXS intensity were used in subsequent calculations.

At the outset, five background corrected intensity values near the origin were fitted to the Gauss curve (Vonk ${ }^{21}$ )

$$
\tilde{I}(x \rightarrow 0)=p \cdot \exp \left(-q x^{2}\right)
$$

by least square technique. The values of constants $p$ and $q$ were obtained as 614 and 184 , respectively. Taking these values of $p$ and $q$, the scattering curve was extrapolated to $x=0$. Each extrapolated point is indicated by the symbol $\triangle$ in Figure 2.

It may be mentioned here that the method of extrapolation has little effect. on the relevant part of the correlation function; neither the position nor the height of the first maximum of the one-dimensional correlation function is much affected.

The two integrals in eq 4 were calculated by numerical integration using Simpson's one third rule and the value of $R$ was found to be $65 \times 10^{-5} \AA^{-2}$. The small but positive value of $R$ indicates that the electron density gradient at the phase boundary is finite suggesting the sample to be a non-ideal two-phase system.

Using eq 6, the three-dimensional corre- 
T. Misra et al.

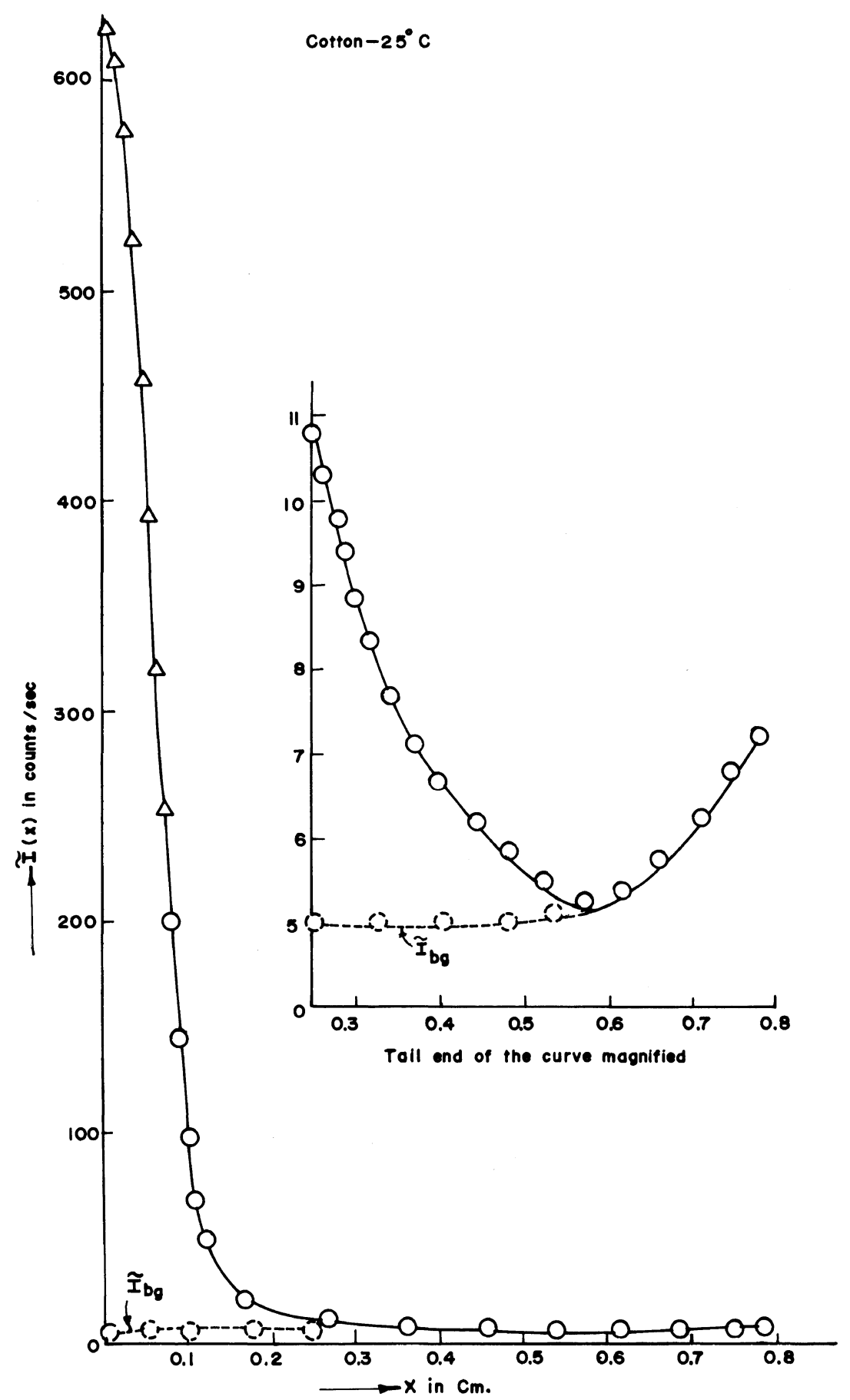

Figure 2. Smeared out scattering curve and background correction curve $\tilde{I}_{\mathrm{bg}}$. 


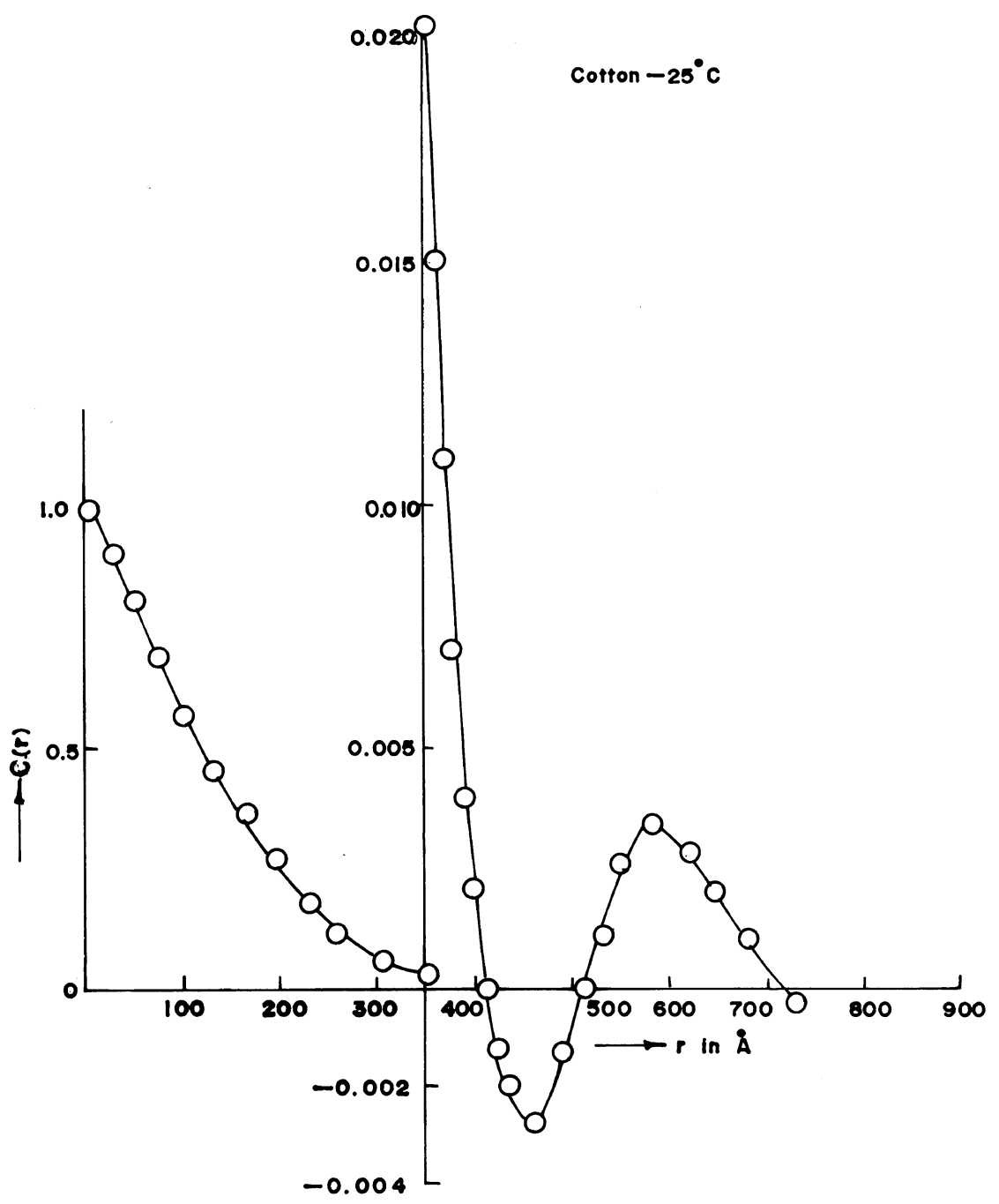

Figure 3. The curve showing the value of three-dimensional correlation function $C(r)$ against ' $r$ ' values. Portion of the curve beyond $r=350 \AA$ magnified.

lation function $C(r)$ was computed for various values of $r$ and is shown in Figure 3 and the portion beyond $r=350 \AA$ is shown on a magnified scale. The slopes of $C(r)$ at different points $r$ were computed by numerical differentiation taking five consecutive points two from each side of the point $r$, (where the slope is found by the central difference method) with a constant interval of $1 \AA$.

The values of $-(4 / R(\mathrm{~d} C(r) / \mathrm{d} r))$ vs. $r$ are plotted in Figure 4. In this figure, a straight line equidistant from both axes is drawn and the point of intersection of this line with the curve indicates the value of $E_{1}$ (using eq 5) to be $33 \AA$

As shown by Jones ${ }^{22}$ by an electron micrograph, cellulose in alga has a layer structure. We assume that the cellulose fibrils in cotton are arranged in layers giving a lamellar structure. That is why a one-dimensional correlation function applicable to a layer structure was calculated for cotton. 


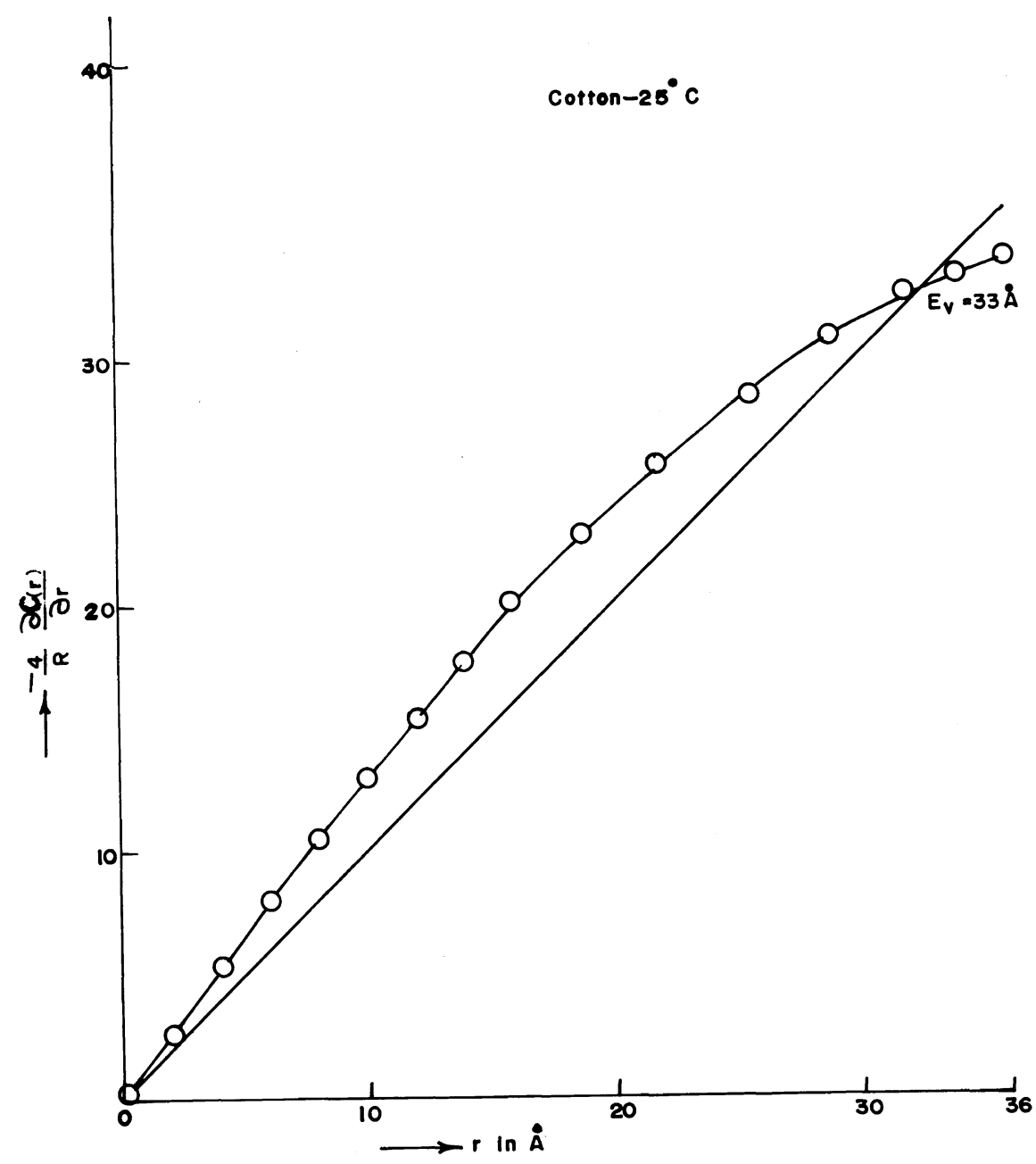

Figure 4. The cruve showing the various values of $-((4 / R) /(\partial C(r) / \partial r))$ against ' $r$ ' values.

Using eq 7 the one-dimensional correlation function $C_{1}(y)$ was calculated for various values of $y$. The values of $C_{1}(y)$ are plotted against $y$ in Figure 5 and the curve beyond $y=$ $450 \AA$ is shown on a magnified scale. The value of $D$, the periodicity transverse to the layer, was found equal to $517 \AA$ which is given by the position of first subsidiary maximum in Figure 5.

Using eq 9 the value of $S / V$ the specific inner surface was obtained as equal to $3.9 \times$ $10^{-3} \AA^{-1}$.

The values of the slopes $\mathrm{d} C_{1}(y) / \mathrm{d} y$ were calculated at various points. The slopes are constant and equal to $-0.13 \times 10^{-1} \AA^{-1}$ for various values of $y>E$ in the range $y=33 \AA$ to $y=100 \AA$. The value of $(\Delta \eta)^{2} /\left\langle\eta^{2}\right\rangle$ was obtained as equal to 6.72 from eq 8 .

From eq 10 the value of the product $\left(\phi_{1}, \phi_{2}\right)$ was found to be 0.1676 . Assuming $\phi_{1}+\phi_{2}=1$, the values of $\phi_{1}$ and $\phi_{2}$ were obtained as $78.7 \%$ and $21.3 \%$, respectively.

Moreover, the value of $\mathrm{d}^{2} C_{1}(y) / \mathrm{d} y^{2}$ at the origin was calculated as $-64.79 \times 10^{-5} \AA^{-2}$ which is nearly equal to three times the value of $\mathrm{d}^{2} C(r) / \mathrm{d} r^{2}$ at the origin following eq $8 . \mathrm{a}$ 


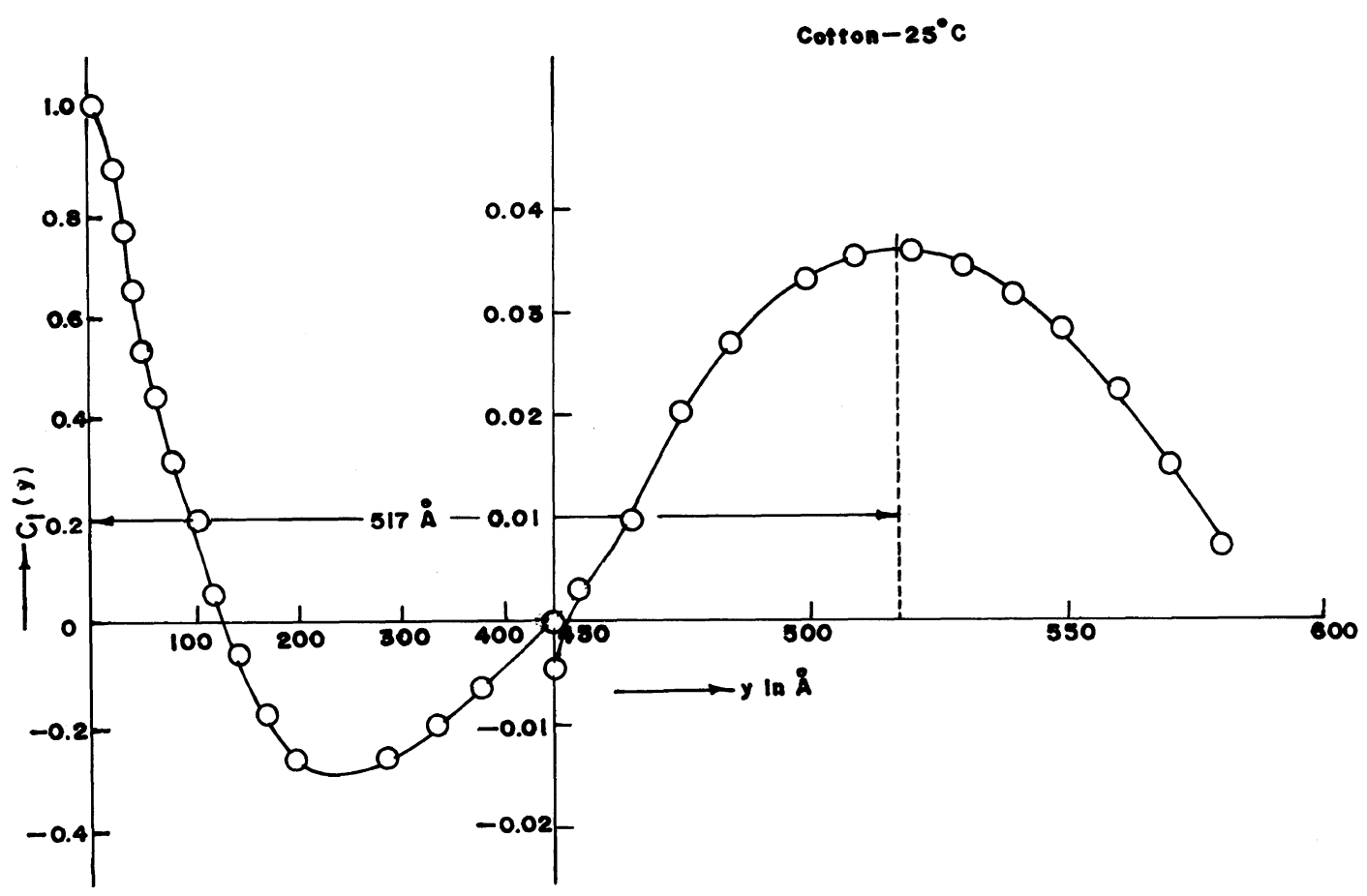

Figure 5. The curve showing the values of one-dimensional correlation function $C_{1}(y)$ against ' $y$ ' values. Curve beyond $y=450 \AA$ magnified.

given by Vonk. ${ }^{1}$

Using eq 11 , the values of the transversal lengths $\bar{l}_{1}$ and $\bar{l}_{2}$ were estimated

$$
I_{1}=814 \AA \text { and } I_{2}=220 \AA .
$$

Following eq 12 , the range of inhomogeneity $\bar{l}_{\mathrm{r}}$ was found out to be $173 \AA$.

The length of Coherence was calculated from eq 13 and was obtained as $l_{\mathrm{c}}=266 \AA$.

The value of $2 E / D$ the volume fraction of the transition layer was calculated as $11.3 \%$.

The Ruland plot of $\tilde{I}(x \rightarrow \infty) \cdot x$ vs. $x^{-2}$ shown in Figure 6, gives a straight line at the tail end of the scattering curve. The slope and intercept of this line were 0.256 and -0.8 , respectively, so that the value of the width of the transition layer $E_{2}$, by Ruland ${ }^{2}$ method was calculated as $30 \AA$. The standard deviation of intensities $\sigma(\sqrt{\tilde{I}})$ at the tail end of the SAXS pattern was calculated to be 0.18 indicating the correctness of the data $\left(V_{0 n k}{ }^{23}\right)$.

\section{CONCLUSIONS AND DISCUSSION}

Cotton belongs to a Non-Ideal Two-Phase Structure

As mentioned earlier for an ideal two-phase structure, the intercept of the Ruland plot vanishes leading to application of Porod's law. When this intercept is negative, the system is considered to be a non-ideal two-phase system and the magnitude of the negative intercept provides a measure of deviation from an ideal to non-ideal structure, i.e., deviation from Porod's law. Whenever the intercept becomes positive the conclusion is ambiguous, either the system does not belong to a two-phase structure or the dimensions of the scattering particles are too small to be studied by the SAXS method.

For cotton, the intercept of the Ruland plot in Figure 6 has a finite negative value. The finite negative value of intercept shows that 


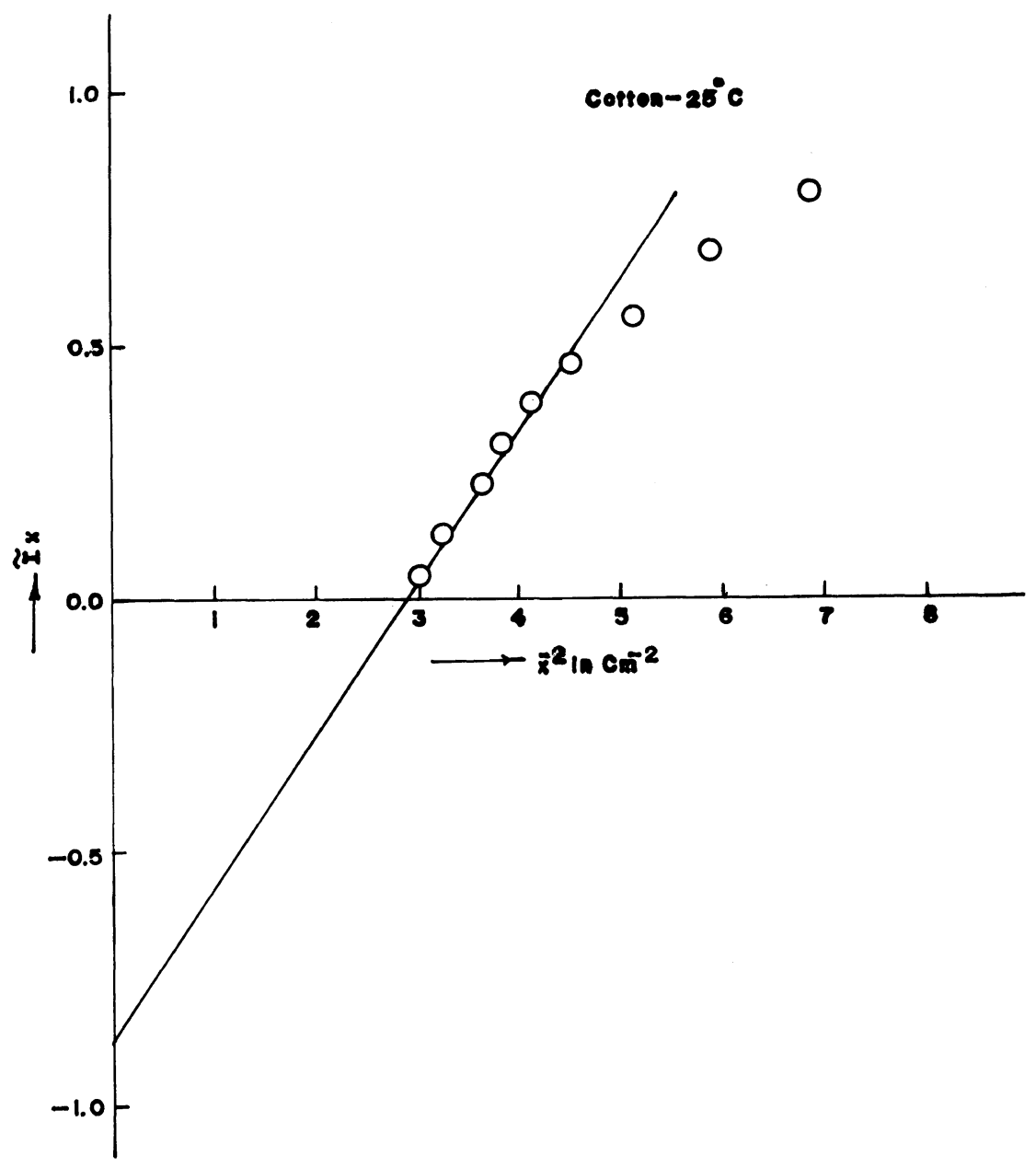

Figure 6. The plot $\tilde{I} x v s .1 / x^{2}$ values.

cotton is a non-ideal two-phase system. The surface between the void and material layers in the structure of cellulose in cotton is not smooth and has a transition layer. The width of the transition layer as determined by the method of Vonk is $E_{1}=33 \AA$ and by the method of Ruland, $E_{2}=30 \AA$. The values obtained by these two methods are approximately equal showing the procedure in this analysis to be correct.

The value of $D$, the periodicity transverse to the layer, is $517 \AA$.

The values of $S / V=3.9 \times 10^{-3} \AA^{-1}, \phi_{1}=$ $0.79, \phi_{2}=0.21, \quad l_{1}=814 \AA, \quad I_{2}=220 \AA, \quad l_{\mathrm{r}}=$
$173 \AA, l_{\mathrm{c}}=266 \AA$, and $2 E / D=11.3 \%$ throw some light on the structural aspects of a sample of cellulose. The lamellar model based on the above findings is shown in Figure 7.

The value of $C(r)$ initially remains positive and goes to zero for a dilute system at large values of $r$ while in a densely packed system, it becomes negative at large values of $r$ (Guinier and Fournet ${ }^{24}$ ). But in the case of a non-ideal two-phase system, the $C(r)$ function oscillates at large values of $r$ (Figure 3). 


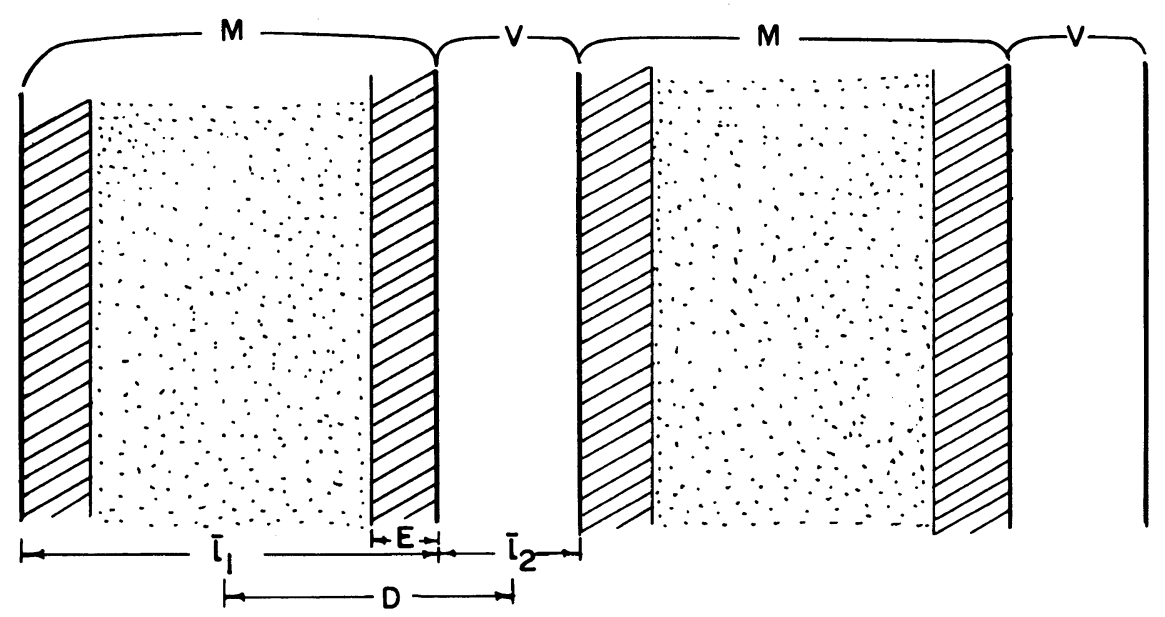

Figure 7. Lamellar model, not to scale: M, matter; V, void; D, periodicity transverse to the layer; E, width of transition layer.

\section{REFERENCES}

1. C. G. Vonk, J. Appl. Cryst, 6, 81 (1973).

2. W. Ruland, J. Appl. Cryst., 4, 70 (1971).

3. O. Kratky, In small angle X-ray scattering, $\mathbf{H}$. Brumberger, Ed., Gordon and Breach, New York, N. Y., 1967.

4. "Encyclopedia of Chemical Technology," 2nd ed, Vol. 6, 1965, p 399.

5. "Encyclopedia of Chemical Technology," 2nd ed, Vol. 4, 1964, p 598.

6. P. Krishnamurti, Ind. J. Phys., 5, 473 (1930).

7. H. Mark, "Physik and Chemic der Zellulose," Springer, Berlin, 1932, p 139.

8. S. B. Hendricks, Z. Kristollogr. Mineral, 83, 503 (1932).

9. G. Porod, Kolloid Z., 124, 83 (1951).

10. G. Porod, Kolloid Z., 125, 51 (1952).

11. S. C. Roy, Text Res. J., 30, 451 (1960).

12. T. Ratho and N. C. Sahu, Indian J. Pure Appl. Phys., 3(5), 63 (1965).
13. G. Porod, Kolloid Z. Z. Polym., 133, 16 (1953).

14. T. Ratho and N. C. Sahu, Kolloid Z. Z. Polym., 236, 43 (1970).

15. T. Misra, K. C. Patra, and T. Patel, Colloid Polym. Sci., 262, 611 (1984).

16. J. Mering and D. Tchoubar, J. Appl. Cryst., 1, 153 (1965).

17. T. Gerber, G. Walter, and R. Krenold, J. Appl. Cryst., 15, 143 (1982).

18. G. Kortleve and C. G. Vonk, Kolloid Z. Z. Polym., 225, 124 (1968).

19. P. Mittelbach and G. Porod, Kolloid Z. Z. Polym., 202, 40 (1965).

20. G. Kortleve, C. A. P. Tuynman, and C. G. Vonk, Polym. Sci., A, 2247, 851 (1972).

21. C. G. Vonk, J. Appl. Cryst., 8, 340 (1975).

22. D. M. Jones, Adv. Carbihydr. Chem., 19, 219 (1964).

23. C. G. Vonk, J. Appl. Cryst., 4, 340 (1971).

24. A. Guinier and G. Fournet, "Small-angle scattering of X-rays," John Wiley \& Sons, Inc., New York, N. Y., 1955, p 79. 